\title{
FUNGOS ASSOCIADOS A SEMENTES HÍBRIDAS DE DENDEZEIRO
}

\author{
Alex Queiroz Cysne'; Wanderlei Antônio Alves de Lima²; Maria Geralda de Souza3. \\ 'Embrapa Amazônia Ocidental, Manaus, Amazonas, Brasil, alex.cysne@embrapa.br \\ 2Embrapa Amazônia Ocidental, Manaus, Amazonas, Brasil, wanderlei.lima@embrapa.br \\ 3Embrapa Amazônia Ocidental, Manaus, Amazonas, Brasil, maria.geralda@embrapa.br
}

\begin{abstract}
RESUMO: O conhecimento dos principais fungos que possam comprometer a qualidade das sementes torna-se indispensável no estabelecimento de áreas de cultivo de híbridos de dendezeiro, de forma a evitar a redução na produção e o aumento de custos. Assim, objetivou-se identificar os principais fungos associados a sementes híbridas intraespecíficas e interespecíficas de dendezeiro. Os lotes de sementes híbridas foram submetidos ao método de incubação em papel filtro (Blotter Test). O delineamento utilizado foi o inteiramente casualizado com 10 repetições. Foram detectados 22 fungos, divididos em saprófitas e potencialmente patogênicos. Destes destacam-se Thielaviopsis sp. e Rhizoctonia sp. como os mais frequentes encontrados em sementes híbridas interespecíficas e intraespecíficas, respectivamente.
\end{abstract}

PALAVRAS-CHAVE: Elaeis guineensis, Micofauna, patologia de sementes.

\section{FUNGUS ASSOCIATED WITH HYBRID SEEDS OF OIL PALM}

ABSTRACT: The knowledge of the main fungi that can compromise the quality of the seeds becomes indispensable in the establishment of areas of cultivation of hybrids of oil palm, in order to avoid a reduction in production and an increase in costs. Thus, the objective was to identify the main fungi associated with intraspecific and interspecific hybrid seeds of oil palm. The hybrid seed lots were submitted to the Blotter Test. The design was completely randomized with 10 repetitions. 22 fungi were detected, divided into saprophytes and potentially pathogenic. These stand out Thielaviopsis sp. and Rhizoctonia sp. as the most frequently found in interspecific and intraspecific hybrid seeds, respectively.

KEYWORDS: Elaeis guineensis, Mycofauna, Seed pathology. 


\section{HONGOS ASOCIADOS CON LAS SEMILLAS HÍBRIDAS DE PALMA DE ACEITE}

RESUMEN: El conocimiento de los principales hongos que pueden comprometer la calidad de las semillas es indispensable en el establecimiento de áreas de cultivo de híbridos de palma aceitera, para evitar la reducción en la producción y el aumento de costos. Así, se objetivó identificar los principales hongos asociados a semillas híbridas intraespecíficas e interespecíficas de palma aceitera. Los lotes de semillas híbridas se sometieron al método de incubación en papel filtro (Blotter Test). El delineamiento utilizado fue el completamente casualizado con 10 repeticiones. Se detectaron 22 hongos, dividido en saprófitos y potencialmente patógenos. Estos destacan Thielaviopsis sp. y Rhizoctonia sp. como el más común que se encuentra en las semillas híbridas interespecíficas e intraespecíficas, respectivamente.

PALABRAS CLAVE: Elaeis guineensis, Micofauna, Patología de semillas.

O dendezeiro (Elaeis guineensis) caracteriza-se como a oleaginosa de maior produção e consumo mundial de óleo (USDA, 2013). A hibridação intraespecífica do dendezeiro ou interespecífica do dendê com a espécie americana, o caiaué (E. oleífera) tem como finalidade desenvolver cultivares mais produtivas, de menor porte e resistentes a doenças, principalmente ao distúrbio denominado amarelecimento fatal (CUNHA; LOPES, 2010).

Para o estabelecimento de áreas de cultivo de híbridos de dendê, são necessárias sementes com qualidade fisiológica e sanitária comprovada, uma vez que, sementes provenientes de campos de produção podem transportar diversas espécies de fungos, os quais, respondem por uma infinidade de enfermidades e danos que afetam as sementes (GALLI et al., 2007). Desta maneira, torna-se indispensável o conhecimento dos principais microrganismos com potencial patogênico que comprometa a qualidade das sementes e mudas.

Assim, devido à escassez de informações quanto à qualidade sanitária de sementes desta espécie, este estudo objetivou identificar os 
principais fungos associados a sementes híbridas intra e interespecíficas de dendê.

Este trabalho foi conduzido no Campo Experimental do Rio Urubu localizado no município de Rio Preto da Eva, AM $\left(02^{\circ} 35^{\prime} S, 59^{\circ} 28^{\prime} \mathrm{W}\right.$, altitude de 200m). As sementes híbridas intraespecíficas (Deli x La Mé) e interespecíficas (Elaeis oleifera $\times E$. guineensis), foram produzidas e coletadas no ano de 2014. Os cachos provenientes dos genitores ascendentes de cada híbrido foram colhidos no ponto de maturação fisiológica, em torno de 150 dias após a polinização. Após despiguetamento dos cachos com auxílio de uma machadinha para retirada da ráquis, as espiguetas foram dispostas em caixas plásticas onde permaneceram por três dias para a fermentação.

Os frutos foram separados das espiguetas e submetidos ao beneficiamento (remoção do mesocarpo, 48 horas de secagem na temperatura ambiente e armazenadas em sala fria a $21^{\circ} \mathrm{C}$ durante 15 dias)
(CYSNE et al., 2015). Dos lotes iniciais de sementes, foram utilizadas 1200 sementes, sendo 600 sementes para cada híbrido.

Após beneficiamento foi analisada a qualidade sanitária das sementes por meio do método do papel de filtro, onde as sementes foram incubadas em substrato de papel de filtro (Blotter test), que consistiu em dispor as sementes em placas do tipo gerbox, sobre duas folhas de papel do tipo germitest, previamente umedecidas com água destilada até saturação. Em seguida, as caixas foram acondicionadas em sala climatizada, com temperatura de $25^{\circ} \mathrm{C}$, por sete dias, quando então, efetuou-se a observação das estruturas fúngicas com auxílio de microscópio estereoscópico e óptico (BRASIL, 2009). A identificação da população fúngica foi realizada por meio de comparação com as características descritas em literatura específica (BARNETT; HUNTER, 1998).

Para cada híbrido de dendezeiro se utilizou o delineamento inteiramente 
casualizado, tendo como tratamentos

22 fungos. Foram utilizadas 10 repetições, sendo 10 sementes por parcela (gerbox). Os dados de incidência (proporção de fungos por parcela) foram submetidos à análise de variância após transformação em $\sqrt{x+1,0}$, uma vez que a pressuposição de normalidade do desvio (cijk) não foi satisfeita pelo teste de Shapiro-Wilk. A análise de variância obedeceu ao modelo estatístico: $Y=\mu+F+\varepsilon$, em que $Y$ é o valor observado dos fungos; $\mu$ é a média da população, F; é o efeito de fungos e $\varepsilon$; erro. As médias foram comparadas pelo teste de Tukey $(p<0,05)$. As análises estatísticas foram realizadas pelo programa SISVAR (FERREIRA, 2008).

Os resultados revelaram que a micofauna presente nas sementes analisadas é constituída de fungos potencialmente patogênicos e de fungos saprófitas (Tabela 1). Dentre os fungos identificados, destaca-se estatisticamente como os mais frequentes Rhizoctonia sp., Fusarium sp. e Lasiodiplodia sp. para o híbrido intraespecífico, com incidências de 31,2\%, 27,9\% e 18,5\%, respectivamente. Já no híbrido interespecífico Fusarium sp. (24,4\%), Thielaviopsis sp. (41,9\%) e Lasiodiplodia sp. (11,3\%) destacam-se como os mais significativamente presentes.

Os fungos Aspergillus sp., Cercospora sp., Rhizopus sp., Ceratocystis sp., Geotrichum sp., Cephalosporium sp., Chalariopsis sp., Graphium sp., Choanephora sp., Genicularia sp., Curvularia sp., Verticillium sp., Epicoccum sp., Phyllosticta sp. e Pythium sp., apresentaram uma incidência inferior a 3\% sobre as sementes de ambos os híbridos.

O comportamento apresentado por estes gêneros o caracteriza como fungos contaminantes, uma vez que, não foram bem-sucedidos em sua proliferação nas sementes. $\bigcirc$ que segundo Machado (1988), são microrganismos associados a tecidos sem atividade enzimática, seja ele, superficial ou interno. Ainda segundo o 
mesmo autor, a infecção somente internos da semente com atividade ocorre quando há uma associação vital. entre o microrganismo e os tecidos

Tabela 1. Ocorrência fúngica (\%) em sementes provenientes de lotes de sementes híbridas intraespecíficas e híbridas interespecíficas de dendezeiro. Embrapa Amazônia Ocidental. Rio Preto da Eva, AM. 2014.

\begin{tabular}{|c|c|c|}
\hline \multirow{2}{*}{ Fungos } & Intraespecífico & Interespecífico \\
\hline & \multicolumn{2}{|c|}{ \% de ocorrência } \\
\hline Rhizoctonia sp. & $31,2 a^{*}$ & $3,9 d$ \\
\hline Fusarium sp. & 27,9 a & $24,4 b$ \\
\hline Aspergillus sp. & $0,7 d$ & 0,6 de \\
\hline Cercospora sp. & $0,3 d$ & 0,5 de \\
\hline Rhizopus sp. & $2,9 \mathrm{~cd}$ & $1,9 d$ \\
\hline Paecilomyces sp. & $5,1 c$ & $0,2 \mathrm{e}$ \\
\hline Thielaviopsis sp. & $2,6 \mathrm{~cd}$ & 41,9 a \\
\hline Ceratocystis sp. & $0,3 d$ & 0,2 e \\
\hline Geotrichum sp. & $1,2 \mathrm{~cd}$ & $0,2 \mathrm{e}$ \\
\hline Trichoderma sp. & $2,3 \mathrm{~cd}$ & $6,1 \mathrm{~cd}$ \\
\hline Cephalosporium sp. & $0,2 d$ & 0,0 e \\
\hline Chalariopsis sp. & $0,3 d$ & 0,0 e \\
\hline Penicillium sp. & $2,2 \mathrm{~cd}$ & $5,8 \mathrm{~cd}$ \\
\hline Graphium sp. & $0,2 d$ & 0,0 e \\
\hline Choanephorasp. & $0,5 d$ & $0,0 \mathrm{e}$ \\
\hline Genicularia sp. & $0,7 d$ & $0,1 \mathrm{e}$ \\
\hline Curvularia sp. & $0,4 d$ & $0,2 \mathrm{e}$ \\
\hline Verticillium sp. & $1,3 \mathrm{~cd}$ & $1,1 \mathrm{de}$ \\
\hline Epicoccum sp. & $0,4 d$ & 0,0 e \\
\hline Phyllosticta sp. & $0,0 d$ & $0,3 \mathrm{e}$ \\
\hline Pythium sp. & $0,0 d$ & $0,3 \mathrm{e}$ \\
\hline Lasiodiplodia sp. & $18,5 b$ & $11,3 c$ \\
\hline
\end{tabular}

* Letras iguais não diferem estatisticamente pelo teste de Tukey $(p>0,05)$

Para os fungos saprófitas estes fungos estão comumente Trichoderma sp. e Penicillium sp. foi associados a sementes beneficiadas e observada uma baixa ocorrência nos armazenadas inadequadamente. dois híbridos. Para Dhingra et al. (1980), Particularmente para estas sementes, 
Mora et al. (2007), relatam que o fungo Penicillium sp. não só é capaz de deteriorar o potencial germinativo destas sementes, como pode afetar o processo de germinação causando a morte do embrião, reduzindo de certa forma, a emergência de plantas em pré-viveiro.

Dos fungos potencialmente patogênicos às sementes de dendezeiro, foram identificados os gêneros Fusarium sp., Rhizoctonia sp., Lasiodiplodia sp. e Thielaviopsis sp., os quais, apresentaram incidências estatisticamente significativas, seja para os dois híbridos de dendezeiro ou somente para um deles. Flood et al. (1990) encontraram cinco espécies de Fusarium spp. associadas as sementes desta espécie, as quais algumas destas podem ocasionar podridões radiculares de plântulas ou da flecha em viveiro. Barcelos et al. (2001) destacam que o Lasiodiplodia sp. e Rhizoctonia sp. causam doenças no dendezeiro em viveiro. Segundo os autores, o Lasiodiplodia sp. é responsável pela antracnose, doença que causa manchas foliares e pode comprometer o processo fotossintético das mudas, e Rhizoctonia sp. pode causar lesões nas folhas jovens, avançando a uma rápida seca após abertura da folha flecha. E Eziashi et al. (2006) destaca uma espécie de Thielaviopsis sp. como sendo o estágio imperfeito de Ceratocystis paradoxa, patógeno que causa a doença podridão negra em sementes germinadas.

Os resultados mostram que a micofauna associada às sementes é composta por vários gêneros, que podem interferir na germinação de sementes e produção de mudas, refletindo na qualidade das sementes produzidas. Assim, este trabalho demonstra a importância da manutenção da qualidade fitossanitária destas sementes.

A partir dos resultados é possível concluir que; (1) dentre os fungos identificados, Thielaviopsis sp. e Rhizoctonia sp. foram os mais frequentes encontrados em sementes híbridas interespecíficas e 
intraespecíficas de dendezeiro, respectivamente. (2) A maior parte da micofauna presente nas sementes híbridas de dendezeiro é formada por fungos contaminantes saprófitas. (3) Fusarium sp., Rhizoctonia sp., Lasiodiplodia sp. e Thielaviopsis sp. são os fungos potencialmente patogênicos mais presentes em sementes híbridas de dendezeiro.

\section{AGRADECIMENTOS}

À Fundação de Amparo à Pesquisa do Estado do Amazonas (FAPEAM) pelo apoio financeiro.

\section{REFERÊNCIAS}

BARCELOS, E.; RODRIGUES, M. R. L.; SANTOS, J. A.; CUNHA, R. N. V. Produção de mudas de dendezeiro na Amazônia. Manaus: Embrapa Amazônia Ocidental, 2001. 12 p. (Embrapa Amazônia Ocidental, Circular técnica, 08).

BARNETT, H. L.; HUNTER, B. B. Illustrated genera of imperfect fungi. Ed. 4. Minnesota. The American Phytopathological Society. 218p. 1998.

BRASIL. Ministério da Agricultura, Pecuária e Abastecimento. Secretaria de Defesa Agropecuária. Manual de análise sanitária de sementes. Brasília, 2009. 200 p.

CUNHA, R. N. $V_{. ;}$LOPES, R. BRS Manicoré: Híbrido Interespecífico entre - Caiaué e o Dendezeiro Africano Recomendado para Áreas de Incidência de Amarelecimento-Fatal. Embrapa Amazônia Ocidental. Comunicado Técnico, n. 85. 4p. 2010.

CYSNE, A. Q.; SOUZA, M. G.; LIMA, W. A. A. Fungos associados a sementes híbridas interespecíficas de dendê em função da assepsia e do beneficiamento. Revista de Ciências Agrárias, v. 58, n. 4, p. 372-378, out./dez. 2015.

DHINGRA, O. D.; MUCHOVEJ, J. J.; CRUZ FILHO, J. Tratamento de sementes (Controle de patógenos). Viçosa: UFV, Imprensa Universitária, 121p., 1980

EZIASHI, E. I.; UMA, N. U.; ADEKUNLE, A. A.; OMAMOR, I. B. Biological control of Ceratocystis paradoxa causing Black seed rot in oil palm sprouted seeds by Trichoderma species. Pakistan Journal of Biological Science, v. 9, n. 10, p. 19871990. 2006.

FERREIRA, D. F. SISVAR: um programa para análises estatísticas e ensino de estatística. Revista Symposium, LavrasMG, v. 6, p. 36-41. 2008.

FLOOD, J.; MEPSTED, R.; COOPER, R. M. Contamination of oil palm pollen and seeds by Fusarium spp. Mycological Research, v. 94, n. 5, p. 708-709. 1990. 
GALLI, J. A.; PANIZI, R. C.; VIEIRA, R. D. Sobrevivência de patógenos associados a sementes de soja armazenadas durante seis meses. Revista Brasileira de Sementes, v. 29, n. 2, p. 205-213. 2007.

MACHADO, J. C. Patologia de sementes: fundamentos e aplicações. Brasília: MEC, 107p. 1988.

MORA, S.; CHINCHILLA, C.; SÁNCHEZ, A.; ESCOBAR, R. Innovación en los procesos para mejorar la calidad de las semillas germinadas y de lãs plántulas de palma aceitera. Palmas, v. 28, n. Especial, Tomo 1, p. 265-272, 2007.

USDA. United States Departament of Agriculture. Oil Seeds: World Markets and Trade. Foreign Agricultural Service. Circular Series. FOP 07, July 2013. Disponível em: <http://www.fas.usda.gov/oilseeds/def ault.asp>. Acesso em 23 de julho de 2013. 